\title{
Construção e validação de afirmativas de diagnósticos de enfermagem para adolescentes hospitalizados
}

\author{
Construction and validation of nursing diagnosis statements for adolescent inpatients
}

Construcción y validación de nomenclaturas de diagnósticos de enfermería para adolescentes hospitalizados

Daniela Karina Marques ${ }^{1}$ Maria Miriam Lima Nóbrega², Kenya Lima Silva ${ }^{3}$

\section{RESUMO}

Este estudo objetivou desenvolver afirmativas de diagnósticos de enfermagem a partir dos indicadores clínicos das Necessidades Humanas Básicas identificadas em adolescentes hospitalizados, utilizando a Classificação Internacional para a Prática de Enfermagem $\left(\mathrm{CIPE}^{\oplus}\right)$. Trata-se de uma pesquisa exploratório-descritiva, realizada na Clínica Pediátrica de um Hospital Escola. A amostra foi composta por enfermeiras assistenciais e docentes atuantes na Clínica. Após revisão da literatura foram consideradas 33 necessidades, sendo identificados 484 indicadores empíricos para adolescentes hospitalizados, os quais foram validados, resultando em 319 indicadores, sendo 200 nas necessidades psicobiológicas, 116 nas necessidades psicossociais e 3 na necessidade psicoespiritual. Em seguida foram validadas 60 afirmativas de diagnósticos de enfermagem utilizando a CIPE ${ }^{\circledR}$. Conclui-se que o desenvolvimento de afirmativas de diagnósticos de enfermagem para adolescentes hospitalizados contribui para atender de forma mais específica as suas necessidades, bem como para o avanço na implementação da Sistematização da Assistência de Enfermagem no hospital pesquisado.

Descritores: Assistência de Enfermagem; Adolescente Hospitalizado; Processos de Enfermagem; Diagnóstico de Enfermagem.

\section{ABSTRACT}

The objective of this study was to develop nursing diagnosis statements based on the clinical indicators regarding the Basic Human Necessities among adolescent inpatients, using the International Classification for Nursing Practice (ICNP ${ }^{\circledR}$ ). This exploratorydescriptive study was performed at a pediatric clinic of a teaching hospital. The sample consisted of clinical nurses and faculty working at the clinic. Following a literature review, 33 necessities were considered, and 484 empirical indicators were identified regarding adolescent inpatients, which were then validated, resulting in 319 indicators: 200 for psychobiological necessities, 116 for psychosocial necessities, and 3 forpsychospiritual necessities. Next, 60 nursing diagnosis statements were validated using the ICNP ${ }^{\circledR}$. In conclusion, the development of diagnosis statements for adolescent inpatients helps to meet their needs more specifically, as well as advancing the implementation of nursing care systematization in the studied hospital.

Descriptors: Nursing Care; Adolescent, Hospitalized; Nursing Process; Nursing Diagnosis.

\section{RESUMEN}

Se objetivó desarrollar nomenclaturas de diagnósticos de enfermería en base a los indicadores clínicos de las Necesidades Humanas Básicas identificadas en adolescentes hospitalizados, utilizando la Clasificación Internacional para la Práctica de Enfermería (CIPE ${ }^{\circledR}$ ). Investigación exploratorio-descriptiva, realizada en la Clínica Pediátrica de un Hospital Escuela. Muestra compuesta por enfermeras asistenciales y docentes actuantes en la Clínica. Luego de revisarse la literatura, fueron consideradas 33 necesidades, identificándose 484 indicadores empíricos para adolescentes hospitalizados, los cuales fueron validados, resultado en 319 indicadores, correspondiendo 200 a necesidades psicobiológicas, 116 a necesidades psicosociales y 3 a necesidades espirituales. A continuación, fueron validadas 60 nomenclaturas de diagnósticos de enfermería utilizando la CIPE $^{\circledR}$. Se concluye en que el desarrollo de nomenclaturas de diagnósticos de enfermería para adolescentes hospitalizados contribuye para atender más específicamente sus necesidades, así como para avanzar en la implementación de la Sistematización de la Atención de Enfermería en el hospital investigado.

Descriptores: Atención de Enfermería; Adolescente Hospitalizado; Procesos de Enfermería; Diagnóstico de Enfermería.

\footnotetext{
${ }^{1}$ Enfermeira, Mestre em Enfermagem. Enfermeira assistencial do Hospital Universitário Lauro Wandeley da Universidade Federal da Paraíba (UFPB). Docente da FACENE. João Pessoa, PB, Brasil. E-mail: danielaantao@hotmail.com.

${ }^{2}$ Enfermeira, Doutora em Enfermagem. Professora Associada da UFPB. João Pessoa, PB, Brasil. E-mail: miriam@ccs.ufpb.br.

${ }^{3}$ Enfermeira, Doutora em Enfermagem. Professora Assistente da UFPB. Enfermeira assistencial do Hospital Universitário Lauro Wandeley da UFPB. João Pessoa, PB, Brasil. E-mail: kenya.lima@ig.com.br.
} 


\section{INTRODUÇÃO}

A adolescência é compreendida como um período extremamente relevante dentro do processo de crescimento e desenvolvimento humano em que ocorrem diversas transformações psicobiológicas, psicossociais e psicoespirituais, resultando em necessidades específicas para esta fase da vida.

Isto faz com que as concepções e práticas de cuidado ao adolescente devam ser constituídas com base em necessidades detectadas buscando transformações focalizadas nos aspectos da humanização, em consonância com as necessidades evidenciadas( ${ }^{(1)}$.

A assistência ao adolescente tem aumentado acentuadamente nas últimas décadas, possibilitando a ampliação de conhecimentos relativos a esse grupo etário e, consequentemente, um atendimento mais eficiente de suas necessidades.

A Política Nacional de Saúde do Adolescente e Jovem preconiza a atenção integral a essa população, e devem ser consideradas as suas necessidades específicas, as características socioeconômicas e culturais da comunidade a qual estão inseridos, bem como as diferenças de gênero, raça e religião. A inserção na agenda pública brasileira da atenção integral à saúde de adolescentes e jovens decorre da mudança de paradigma expressa na concepção ampliada de saúde como direito social e dever do Estado e na doutrina da proteção integral preconizada pelo Estatuto da Criança e do Adolescente (2).

O conhecimento profissional deficiente sobre características específicas de sua clientela assistida dificulta $o$ atendimento adequado de suas necessidades. Para que a Enfermagem solidificasse suas bases científicas desde as décadas de 1950 e 1960, os modelos conceituais e teorias de enfermagem vêm auxiliando na orientação dos profissionais de enfermagem para que eles descrevam e expliquem aspectos da realidade assistencial, contribuindo para que se edifique a tríade teoria, pesquisa e prática na área. Servindo de aportes epistemológicos fundamentais para a construção do saber e a prática profissional(3).

Os modelos conceituais e teorias de Enfermagem foram criados objetivando descrever e caracterizar os componentes dos fenômenos que the são pertinentes, e cujas finalidades são explicar, elucidar e interpretar, ou seja, dizer o significado e o porquê dos fatos e suas relações ${ }^{(4)}$. Representando diferentes maneiras de ver a Enfermagem em perspectivas sobre quem é o cliente, o que é saúde, qual o papel da enfermeira ou das ações, e qual o ambiente no qual a Enfermagem ocorre.

Por meio de maior embasamento teórico-prático, pode-se proporcionar uma assistência direcionada às reais necessidades dos adolescentes. Uma vez atendidas as necessidades básicas, será facilitada a melhora do seu estado saúde/doença, evitando-se o agravamento da sua condição.

Neste estudo, optou-se pela utilização da Teoria das Necessidades Humanas Básicas de Horta, a qual além de ser uma das mais difundidas em todo o país, é uma teoria que se ajusta ao atendimento da clientela escolhida, e se constitui como referencial teórico do Projeto de Sistematização da Assistência de Enfermagem do Hospital Universitário Lauro Wanderley (HULW). Esse projeto busca a integração entre pesquisa de campo e extensão, prevendo a articulação de enfermeiros docentes e assistenciais, com o propósito de desenvolver a sistematização da assistência de enfermagem.

Para contribuir nas considerações relatadas, a enfermeira ao lançar mão da Sistematização da Assistência de Enfermagem (SAE) proporciona um espaço para expressão/captação das necessidades, colaborando para a resolução de problemas de sua competência e articulação com outros setores, profissionais ou estruturas de apoio. As fases Processo de Enfermagem no modelo de cuidar adotado no HULW da Universidade Federal da Paraíba (UFPB) são: histórico de enfermagem, diagnóstico de enfermagem, planejamento da assistência de enfermagem, implementação das intervenções e avaliação, e segue a definição instituída pela resolução 358/2009 do Conselho Federal de Enfermagem ${ }^{(5)}$.

Essas fases do processo de Enfermagem são definidas como: - Histórico de enfermagem (coleta de dados) - processo deliberado, sistemático e contínuo, realizado com o auxílio de métodos e técnicas variadas que têm por finalidade a obtenção de informações sobre a pessoa, família ou coletividade humana. Diagnóstico de Enfermagem - processo de interpretação e agrupamento dos dados coletados na primeira etapa, que culmina com a tomada de decisão e que constituem a base para a seleção das ações ou intervenções com as quais se objetiva alcançar os resultados esperados. 
Planejamento de Enfermagem - é a determinação dos resultados que se espera alcançar; e das ações ou intervenções de enfermagem que serão realizadas. Implementação - realização das ações ou intervenções determinadas na etapa de Planejamento de Enfermagem. Avaliação de Enfermagem - processo deliberado, sistemático e contínuo de verificação de mudanças para determinar se as ações ou intervenções de enfermagem alcançaram o resultado esperado; e de verificação da necessidade de mudanças ou adaptações nas etapas do Processo de Enfermagem(5).

A aplicação do processo de enfermagem vem contribuindo рага o desenvolvimento de sistemas de classificação de enfermagem para os elementos da prática profissional, como a Taxonomia e Diagnóstico de enfermagem da NANDA Internacional, a Classificação de Intervenções de Enfermagem (NIC), a Classificação de Resultados de Enfermagem (NOC), o Sistema Omaha, a Classificação dos Cuidados Domiciliares de Saúde (HHCC), e a Classificação Internacional para a Prática da Enfermagem $\left(\mathrm{CIPE}^{\circledR}\right)$, que tem o seu desenvolvimento e aprimoramento coordenado pelo International Council of Nurse (ICN) (Conselho Internacional de Enfermeiras CIE) ${ }^{(6)}$.

A CIPE $^{\circledR}$ configura-se como um sistema que tem mobilizado as enfermeiras e todo o mundo, a fim de atender o desafio de universalizar a sua linguagem e evidenciar os elementos da sua prática - os diagnósticos, os resultados esperados e as intervenções de enfermagem, como também facilitar o mapeamento cruzado dos termos locais com os termos constantes no Modelo de Sete Eixos, justificando a escolha desse sistema de classificação neste estudo.

Este estudo objetivou desenvolver afirmativas de diagnósticos de enfermagem a partir dos indicadores clínicos das Necessidades Humanas Básicas identificadas em adolescentes hospitalizados, utilizando a Classificação Internacional para a Prática de Enfermagem $\left(\mathrm{CIPE}^{\circledR}\right)$.

\section{METODOLOGIA}

Trata-se de uma pesquisa exploratório-descritiva, que foi desenvolvida na Clínica Pediátrica de um hospital universitário, localizado no município de João Pessoa, Paraíba. Esse hospital é capacitado para aprimoramento dos conhecimentos teórico-práticos de diversos cursos da área de saúde, a fim de prestar uma melhor assistência aos seus usuários, servindo também como campo de pesquisa para diversos profissionais em formação e com a devida supervisão dos profissionais responsáveis.

Participaram do estudo as enfermeiras assistenciais e docentes que atuam na Clínica Pediátrica. A amostra foi composta por 12 profissionais, que por meio da assinatura do Termo de Consentimento Livre e Esclarecido aceitaram participar do estudo. Foram respeitados os aspectos éticos preconizados pela Resolução No 196/96 do Conselho Nacional de Saúde, que regulamenta a pesquisa envolvendo seres humanos $^{(7)}$ e a Resolução COFEN 311/2007 que reformula o código de ética dos profissionais de enfermagem ${ }^{(8)}$. Vale salientar que a pesquisa apenas foi iniciada após receber parecer favorável à sua execução por parte do Comitê de Ética do HULW, conforme o Protocolo 141/2007.

Para o desenvolvimento de afirmativas de diagnósticos, foram executadas duas fases na pesquisa. Na primeira fase foi realizada revisão da literatura em livros, artigos e trabalhos científicos pertinentes ao tema para a identificação dos indicadores empíricos das Necessidades Humanas Básicas em adolescentes hospitalizados a partir do referencial teórico de Horta, que totalizou 484 indicadores empíricos. Esses indicadores foram organizados em um instrumento e passaram por validação das enfermeiras participantes do estudo. Os indicadores deveriam ser considerados relevantes ou não relevantes.

Após o recebimento dos instrumentos, eles foram conferidos quanto ao completo preenchimento; foram classificados por ordem de recebimento para inclusão e construção do banco de dados no Excel for Windows, onde os indicadores que foram classificados como relevantes, receberam peso "1" e não relevantes - peso "0". Os 319 indicadores que possuíram Índice de Concordância (IC) igual ou maior que 0.80 foram validados, e os indicadores com IC inferior a 0.80 não foram considerados para inclusão no histórico de enfermagem.

Na segunda fase ocorreu o desenvolvimento das afirmativas de diagnósticos de enfermagem, a partir dos indicadores empíricos das Necessidades Humanas Básicas que foram validados. Para a construção dessas 
afirmativas foi utilizada a $\operatorname{CIPE}^{\circledR}$ Versão 1.0 , que é uma terminologia combinatória para a prática da Enfermagem, que facilita o mapeamento cruzado de termos locais, classificações e vocabulários existentes.

$\mathrm{O} \mathrm{ClE}^{(6)}$ recomenda que para compor as afirmativas de diagnósticos de enfermagem seja usado o Modelo de Sete Eixos da $\mathrm{CIPE}^{\circledR}$ e as seguintes diretrizes: incluir obrigatoriamente, um termo do eixo Foco e um termo do eixo Julgamento; incluir termos adicionais, conforme a necessidade dos eixos: Foco, Julgamento, Cliente, Localização e Tempo.

Para a elaboração das afirmativas de diagnósticos, levaram-se em consideração os 319 indicadores empíricos validados na primeira fase da pesquisa, estes foram mapeados com os termos da $\mathrm{CIPE}^{\circledR}$ Versão 1.0, para que fosse possível usar os critérios do Conselho Internacional de Enfermeiros na construção das afirmativas. Dos 319 indicadores, 68 foram mapeados como termo do eixo foco e utilizados para a construção dos diagnósticos, que resultaram em 68 afirmativas diagnósticas.

Após a construção das afirmativas de diagnósticos, estas foram submetidas ao processo de validação pelas enfermeiras. Elas deveriam considerar a aplicabilidade, relevância e a frequência de cada diagnóstico para a clientela do estudo, de acordo com as Necessidades Humanas Básicas. Após a validação, o resultado foram 60 afirmativas diagnósticas validadas.

A amostra considerada nessa segunda fase do estudo foi constituída pelas mesmas participantes que validaram o instrumento na primeira fase, por terem conhecimento prévio do conteúdo da pesquisa.

\section{RESULTADOS}

A identificação dos indicadores empíricos das necessidades humanas básicas em adolescentes hospitalizados foi feita tomando como base o que foi proposto por Horta ${ }^{(9)}$, bem como a descrição de Benedet e Bub(10), perfazendo um total de 33 necessidades organizadas nos níveis psicobiológico, psicossocial e psicoespiritual.

Nas necessidades psicobiológicas os indicadores foram direcionados para as seguintes necessidades: oxigenação; hidratação; nutrição; eliminação; sono e repouso; atividade física; sexualidade; cuidado corporal; integridade física; regulação térmica; regulação vascular; regulação neurológica; regulação crescimento celular; regulação hormonal; regulação imunológica; percepção dos órgãos dos sentidos: olfativa, visual, auditiva, tátil, gustativa, dolorosa; segurança física/meio ambiente; terapêutica. Рara as necessidades psicossociais os indicadores foram direcionados para segurança emocional; amor e aceitação liberdade e participação; comunicação; criatividade; educação para a saúde/aprendizagem; gregária; recreação e lazer; espaço; orientação no tempo e no espaço; autorrealização; autoestima, autoconfiança e autorrespeito; autoimagem e atenção. Na necessidade psicoespiritual os indicadores foram referentes à religiosidade/ espiritualidade.

A partir dos indicadores empíricos identificados na literatura, no total de 484, estes foram selecionados por necessidades humanas básicas para adolescentes hospitalizados e 321 indicadores ficaram pertencentes às necessidades psicobiológicas, 152 às necessidades psicossociais e 11 pertencentes à necessidade psicoespiritual. Após o processo de validação dos indicadores empíricos pelas enfermeiras, o resultado foi de 319 indicadores, desses, 200 para as necessidades psicobiológicas, 116 para as necessidades psicossociais e três para a necessidade psicoespiritual.

Esses indicadores ao serem validados por enfermeiras assistenciais e docentes tornaram-se representativos рага a clientela escolhida, constituindo um banco de termos locais mais utilizados na assistência de enfermagem aos adolescentes atendidos na Clínica Pediátrica do HULW.

Utilizando os indicadores relevantes e os critérios do CIE foram construídas e validadas 60 afirmativas de diagnósticos de enfermagem, distribuídas nas Necessidades Humanas Básicas de Horta. Foi considerado, nesse processo, o raciocínio clínico na construção das afirmativas de diagnósticos de enfermagem. As afirmativas de diagnósticos construídas e validadas foram assim distribuídas: 47 nas Necessidades Psicobiológicas, 12 nas Necessidades Psicossociais e uma na Necessidade Psicoespiritual. As afirmativas diagnósticas estão descritas nos Quadros 1 e 2. 
Quadro 1: Relação das afirmativas de diagnósticos de enfermagem para adolescentes hospitalizados que alcançaram IC=0.80, segundo a classificação de Horta de Necessidades Psicobiológicas. João Pessoa, PB, 2008.

\begin{tabular}{|c|c|}
\hline Necessidades Pisicobiológicas & Diagnósticos de Enfermagem \\
\hline Oxigenação ( $n=5)$ & Dispneia; Tosse Produtiva; Taquipneia; Expectoração insuficiente; Coriza. \\
\hline Hidratação (n=5) & Edema; Náusea; Vômito; Retenção de fluidos; Desidratação. \\
\hline Nutrição (n=6) & $\begin{array}{c}\text { Nutrição prejudicada; Obesidade; Desnutrição; Deglutição prejudicada; } \\
\text { Vômito induzido; Intolerância alimentar. }\end{array}$ \\
\hline Eliminação ( $n=5)$ & Constipação; Diarreia; Retenção urinária; Poliúria; Dor durante a micção. \\
\hline Sono e Repouso $(n=1)$ & Sono e repouso prejudicados. \\
\hline Atividade Física $(n=5)$ & $\begin{array}{l}\text { Deambulação prejudicada; Marcha descoordenada; Atividade física } \\
\text { prejudicada; Mobilidade física prejudicada; Intolerância à atividade. }\end{array}$ \\
\hline $\begin{array}{l}\text { Crescimento Celular/ Regulação } \\
\text { Hormonal/ Sexualidade }(n=3)\end{array}$ & $\begin{array}{l}\text { Crescimento e desenvolvimento incompatíveis com a idade; Dismenorreia; } \\
\text { Prática sexual de risco. }\end{array}$ \\
\hline Cuidado Corporal ( $n=3)$ & $\begin{array}{c}\text { Autocuidado prejudicado para __.(especificar); Higiene corporal } \\
\text { prejudicada; Higiene bucal prejudicada. }\end{array}$ \\
\hline Integridade Física $(n=3)$ & $\begin{array}{c}\text { Integridade da pele prejudicada; Prurido (localização); Mucosa oral } \\
\text { prejudicada. }\end{array}$ \\
\hline Regulação Térmica ( $n=3)$ & Hipertermia; Hipotermia; Sudorese intensa. \\
\hline Regulação Vascular ( $n=3)$ & $\begin{array}{l}\text { Pressão arterial alterada; Perfusão periférica diminuída; Rede vascular } \\
\text { periférica prejudicada. }\end{array}$ \\
\hline Orientação no Tempo e no Espaço $(n=1)$ & Desorientação no tempo e no espaço. \\
\hline Regulação Imunológica ( $n=2)$ & Calendário vacinal incompleto; Risco para a infecção. \\
\hline $\begin{array}{l}\text { Percepção dos Órgãos dos } \\
\text { Sentidos/Comunicação }(n=2)\end{array}$ & Percepção sensorial alterada; Dor. \\
\hline
\end{tabular}

Quadro 2: Relação das afirmativas de diagnósticos de enfermagem para adolescentes hospitalizados que alcançaram

IC=0.80, segundo a classificação de Horta das Necessidades Psicossociais e psicoespiritual. João Pessoa, PB, 2008.

\begin{tabular}{|c|c|}
\hline $\begin{array}{c}\text { Necessidades Psicossociais e } \\
\text { Psicoespiritual }\end{array}$ & Diagnósticos de Enfermagem \\
\hline $\begin{array}{c}\text { Autoimagem/ Autoestima, Autoconfiança e } \\
\text { Autorrespeito/ Segurança Emocional. }(n=8)\end{array}$ & $\begin{array}{c}\text { Ansiedade; Depressão; Medo; Imagem corporal prejudicada; } \\
\text { Isolamento social; Interação social prejudicada; Autoestima alterada; } \\
\text { Conflito com a família. }\end{array}$ \\
\hline $\begin{array}{c}\text { Educação para a Saúde/ Aprendizagem/ } \\
\text { Terapêutica }(n=3)\end{array}$ & $\begin{array}{c}\text { Manutenção da saúde alterada; Adesão ao regime terapêutico; Déficit } \\
\text { de conhecimento. }\end{array}$ \\
\hline Recreação e Lazer $(n=1)$ & Atividades de recreação deficientes. \\
\hline Religiosidade/ Espiritualidade $(n=1)$ & Angústia espiritual. \\
\hline
\end{tabular}

\section{DISCUSSÃO}

O enfermeiro ao fundamentar a sua assistência por meio do processo de enfermagem, consegue obter informações relevantes da clientela assistida, faz diagnósticos para as reais necessidades do cliente, elabora plano de cuidados de enfermagem adequado, avalia os resultados alcançados pela assistência, obtendo consequentemente maior qualidade nos cuidados prestados, com maior resolutividade do problema identificado e maior visibilidade da assistência prestada.

Os modelos teóricos têm contribuído muito na prática assistencial da Enfermagem, quando utilizados como referencial para a operacionalização do processo de enfermagem. Isso colabora para que sejam proporcionados caminhos para organizar as informações e os dados dos pacientes, para analisar e interpretar, para cuidar e avaliar os resultados desse cuidado(11).

O processo de enfermagem recebe várias definições de acordo com muitos autores e teorias, sendo que, cada uma delas segue um modelo, sendo fundamentada de acordo com os conceitos, pressupostos e proposições próprias dessas teorias ${ }^{(12)}$. O processo de enfermagem especificamente na fase de diagnóstico de enfermagem auxilia na avaliação da assistência prestada, direciona o cuidado, promove pesquisa e ensino, delimita as funções de enfermagem, estimula o indivíduo a participar do seu tratamento, facilita a comunicação e prioriza o registro da assistência de enfermagem ${ }^{(13)}$.

A CIPE $^{\circledR}$ representa uma ferramenta que produz informações para a tomada de decisão do enfermeiro, 
por meio de uma linguagem unificada e universal. Tratase de uma terminologia combinatória que permite formular diagnósticos de enfermagem, delinear intervenções e identificar resultados esperados ${ }^{(14)}$.

Рага o $\mathrm{CIE}$ a $\mathrm{CIPE}^{\circledR}$ constitui um instrumento de informação, de descrição e provê dados que representam a prática de enfermagem nos sistemas de informação em saúde. Podendo ser utilizada para tornar o exercício da Enfermagem aparente nos sistemas de informação em saúde, como também para descrever e integrar a Enfermagem à pesquisa, educação, administração e gestão, assim como, ao desenvolvimento de políticas dos cuidados de saúde ${ }^{(6)}$. Aumentando a visibilidade da ciência da Enfermagem quando é prestada sua assistência nos diversos campos do saber.

Ao conhecer o perfil de diagnósticos de enfermagem para adolescentes hospitalizados utilizando a teoria das Necessidades Humanas Básicas de Horta com respaldo da CIPE $^{\circledR}$ buscou-se contribuir com uma prática sistematizada de enfermagem e processo de reflexão crítica da atuação profissional, pois a reflexão crítica pode gerar questionamentos e modificações na atuação profissional quando for necessário e, consequentemente, espera-se a melhoria da assistência prestada aos adolescentes atendidos na unidade.

Embora diversas pesquisas já venham discutindo a dificuldade de implementação da Sistematização da Assistência de Enfermagem, esta pesquisa lança um desafio para a prática assistencial da Clínica Pediátrica, bem como para o restante do hospital pesquisado, pois estudos referem diversas dificuldades para a sua efetivação, como: as limitações relacionadas à instituição, a falta de tempo associada ao número reduzido de profissionais e a falta de informatização e no que se refere às limitações dos profissionais aparecem como dificuldades, a pouca experiência e a falta de conhecimento teórico dos profissionais com a operacionalização do processo de enfermagem, além da resistência e da desmotivação(15).

A teoria das Necessidades Humanas Básicas foi desenvolvida baseada na Teoria da Motivação Humana de Maslow, porém utiliza a denominação dos níveis de vida psíquica de Mohana, que são divididos em psicobiológico, psicossocial e psicoespiritual. Esses níveis de vida psíquica possuem componentes inconscientes e que tendem a ser espontâneos ou de necessidades fundamentais da natureza humana ${ }^{(9)}$, os quais serão descritos a seguir.

O nível psicobiológico é uma força que existe nos seres humanos independentemente de leitura, de educação, de cultura e de meio. Surge de forma inesperada para regular todas as funções necessárias a nossa sobrevivência, sendo inconsciente e surgindo sem planejamento prévio, como: respiração, circulação, hidratação, sono, excreção, repouso, sensação de dor, entre outros ${ }^{(16)}$.

Durante o crescimento e desenvolvimento, os indivíduos vivenciam inúmeras e diferentes necessidades, pois a interação de seu potencial genético com o ambiente físico e social thes confere características complexas e dinâmicas. Esse processo não é uniforme nem tampouco contínuo, pois se constitui em diversas fases de rápidas transformações (aceleração recém-nascido; adolescente) e lentas (desaceleração idoso), esse processo depende ainda da variabilidade individual(17).

Particularmente na adolescência ocorre uma transição entre a infância e a idade adulta que se caracteriza por profundas transformações somáticas, psicológicas e sociais. Representa uma das fases mais importantes do ciclo vital, à medida que se completa o período de crescimento e desenvolvimento(1).

O crescimento é aferido com medidas e índices antropométricos com os quais são construídos indicadores, definindo níveis de corte que possibilitem situar o indivíduo dentro de uma faixa aceita como normal, de acordo com um determinado padrão de referência. Já o desenvolvimento é um processo complexo, de transformações contínuas, dinâmicas e progressivas que envolvem o crescimento, a maturação, a aprendizagem e os aspectos psíquicos e sociais ${ }^{(17)}$.

O nível psicossocial é a tendência que todo ser humano tem de conversar, conviver socialmente, afirmar-se perante os outros, tornar visível seu valor, sentir-se querido, amado e aceito pelo outro(16). As dimensões psicológicas e sociais são vivenciadas de maneiras diferentes em cada sociedade, em cada geração e em cada família, sendo singulares até mesmo para cada indivíduo. É nesse contexto de alteração do próprio corpo e também de uma maturação do nível psíquico que o adolescente procura entender quem é, e 
qual é o seu papel na sociedade em que vive, interessando-se por problemas de ordem moral e ética, adotando ideologias para servirem como guia em suas atitudes perante a sociedade.

Em relação à necessidade psicoespiritual, pode-se comentar que se trata de algo muito particular e dependerá da visão de mundo de cada indivíduo, principalmente quando se está enfrentando algum problema, como é especificamente neste estudo a hospitalização na adolescência. Geralmente é diante de crises que os clientes tomam consciência dessa necessidade. Essa "necessidade é inerente aos seres humanos e está vinculada àqueles fatores necessários para o estabelecimento de um relacionamento dinâmico entre a pessoa e um ser ou entidade superior, com o objetivo de sentir bem-estar espiritual"(10).

A importância da construção de um banco de dados que inclua termos que possibilitem a construção de afirmativas de diagnósticos de enfermagem favorece a utilização de uma linguagem comum e, ao mesmo tempo, integra o conhecimento científico e o conhecimento prático da profissão, como também o registro sistemático dos diagnósticos de enfermagem, leva ao aumento de visibilidade e de reconhecimento profissional e, por outro lado, para uma possibilidade concreta de avaliação da prática de enfermagem ${ }^{(18-19)}$.

A Enfermagem só vem conseguindo consolidar-se como ciência e arte porque tem produzido uma linguagem específica que atribui significado aos elementos constitutivos do seu ser, saber e fazer. Assim, a linguagem produzida por determinado campo do saber possibilita a compreensão acerca das representações do pensamento e do mundo, seja como veículo de comunicação ou como instrumento de ação/interação(20).

\section{CONSIDERAÇÕES FINAIS}

A construção e validação de diagnósticos de enfermagem podem ser usadas como ferramentas importantes para aprimorar e direcionar a assistência prestada ao paciente, pautada em conhecimento científico, pois o enfermeiro em sua prática assistencial se depara com inúmeras particularidades para atender diferentes necessidades de seus clientes. Quando são conhecidas as características de sua clientela, por meio de estudos científicos, eles podem contribuir para a melhoria da assistência de enfermagem que é prestada, e para a efetivação da implementação do Processo de Enfermagem na prática clínica.

Considera-se imprescindível que para assistir o paciente é necessário ter conhecimento científico e informações da prática, assim como, da realidade profissional no alcance do melhor resultado possível. Para a Enfermagem contemporânea as teorias de enfermagem são utilizadas na construção efetiva do saber científico. Elas devem ser construídas, testadas e aplicadas à prática assistencial, para cada vez mais transformar e contribuir no embasamento científico e específico da disciplina.

Avaliando a utilização da Teoria de Horta nesta pesquisa considera-se esse referencial importante e aplicável para identificação das necessidades afetadas em adolescentes hospitalizados, para a identificação dos indicadores clínicos e, posteriormente, para a construção de diagnósticos de enfermagem, possibilitando um conhecimento mais aprofundado em relação a essa clientela.

A proposta de trabalhar a Sistematização da Assistência de Enfermagem numa Unidade de Clínica Pediátrica lança inúmeros desafios. Especificamente na Clínica Pediátrica do Hospital em estudo, pois a faixa etária atendida vai de zero a 18 anos. E sabe-se que durante esse intervalo de vida o ser humano passa por diversas e variadas mudanças fisiológicas e psicológicas, sendo necessário que a enfermeira utilize as diferentes abordagens que deverão ser feitas durante 0 atendimento.

Apesar da reconhecida importância do processo de enfermagem e da fase diagnóstica ser considerada uma das mais complexas, por exigir conhecimentos específicos, julgamento crítico e clínico, percebe-se que na prática assistencial, a operacionalização do processo de enfermagem ainda constitui um desafio, devido a fatores inerentes à própria profissão; bem como a fatores relacionados à estrutura organizacional e administrativa da maioria das instituições. Espera-se, portanto que a enfermeira analise de maneira crítica a sua prática profissional, buscando desenvolver competências e conhecimentos que contribuam com a ciência da Enfermagem, bem como, o estudo sirva de avanço na implementação da Sistematização da Assistência de Enfermagem da Clínica Pediátrica, servindo também de modelo para novos estudos e 
contribuições para outras clínicas do hospital pesquisado.

\section{REFERÊNCIAS}

1. Queiroz MVO, Ribeiro EMV, Pennafort VPS. Assistência ao adolescente em um serviço terciário: acesso, acolhimento e satisfação na produção do cuidado. Texto Contexto Enferm. [Internet]. 2010. [cited 2012 jun 20];19(2):291-299. Available from: http://www.scielo.br/pdf/tce/v19n2/10.pdf.

2. Rapozo C. A Política de Atenção Integral à Saúde do Adolescente e Jovem: uma perspectiva de garantia de direito à saúde? Revista Em Pauta. 2009. [cited 2012 mai 08];6(23):117-138. Available from: http://www.epublicacoes.uerj.br/index.php/revistaempauta/article/viewFile/451/ $\underline{549}$.

3. Shaurich D, Crossetti MGO. TM. Produção do conhecimento sobre teorias de enfermagem: análise de periódicos da área, 1998-2007. Esc Anna Nery Rev Enferm 2010. [cited 2012 mai 08];14(1):182-8. Available from: http://www.scielo.br/pdf/ean/v14n1/v14n1a27.pdf.

4. Andrade CA. A Enfermagem não é mais uma profissão submissa. Rev. Bras. Enferm., Brasília 2007;60(16):96-8.

5. Conselho Federal de Enfermagem. Resolução No 358/2009. Sistematização da Assistência de Enfermagem e a implementação do Processo de Enfermagem. Brasília (Brasil): COFEN; 2009. 6. Conselho Internacional de Enfermeiros. CIPE ${ }^{\circledR}$ Versão 1.0: Classificação Internacional para a Prática de Enfermagem. São Paulo: Algol; 2007.

7. Ministério da Saúde; Conselho Nacional de Saúde. Resolução No 196/96 - Normas regulamentadoras de pesquisa envolvendo seres humanos. Brasília (Brasil): Ministério da Saúde; 1996.

8. Conselho Federal de Enfermagem. Resolução COFEN-311/2007. Aprova a Reformulação do Código de Ética dos Profissionais de Enfermagem. Rio de Janeiro (Brasil): COFEN; 2007.

9. Horta WA. Processo de Enfermagem. Rio de Janeiro: Guanabara Koogan;2011.

10. Benedet AS, Bub BC. Manual de diagnóstico de enfermagem: uma abordagem baseada na teoria das necessidades humanas básicas e na classificação de diagnóstico da NANDA. 2.ed. Florianópolis: Bernúncia; 2001.

11. Amante LN, Rossetto AP, Schneider DG. Sistematização da Assistência de Enfermagem em Unidade de Terapia Intensiva sustentada pela Teoria de Wanda Horta. Rev. Enferm. USP. 2009;43(1):54-64.

12. Freitas MC, Queiroz TA, Souza JAV. O Processo de Enfermagem sob a ótica das enfermeiras de uma maternidade. Rev. Bras. Enf. 2007;60(2):207-12.

13. Carpenito-Moyet LJ. Diagnóstico de Enfermagem: aplicação à prática clínica. $11^{\text {a }}$ ed. Porto Alegre: Artmed; 2009.

14. Silva RR, Malucelli A, Cubas MR. Classificações de Enfermagem: mapeamento entre termos do foco da prática. Rev. Bras. Enf. 2008;61(6):835-40.

15. Luiz FF, Mello SMM, Neves ET, Ribeiro AC, Tronco CS. A sistematização da assistência de enfermagem na perspectiva da equipe de um hospital de ensino. Rev. Eletr. Enf. [Internet]. 2010. [cited 2012 mai 09];12(4):655-9. Available from: http://www.fen.ufg.br/fen revista/v12/n4/pdf/v12n4a09.pdf. 16. Mohana J. O mundo e eu. Rio de Janeiro: Agir; 1963. 17. Samico I, Souza MFM, Marinho TMS, Soares TS. Crescimento e Desenvolvimento na infância e adolescência. In: Alves JGB, Ferreira OF, Maggi RS. Fernando Figueira Pediatria Instituto Materno Infantil de Pernambuco (IMIP). 3 ed. Rio de Janeiro: Guanabara Koogan; 2004. 38-57.

18. Coenen A; Kim, TY. Development of terminology subsets using ICNP ${ }^{\circledR}$. International Journal of Medical Informatics. 2010;7(9):5308.

19. Garcia TR, Nóbrega MML. Processo de enfermagem: da teoria à prática assistencial e de pesquisa. Esc Anna Nery Rev Enferm. 2009;13(1):188-93.
20. Garcia TR, Nóbrega MML. Contribuição das teorias de enfermagem para a construção do conhecimento da área. Rev Bras Enferm. 2004;57(2):228-32.

Artigo recebido em 10/08/2011. Aprovado para publicação em 25/04/2012. Artigo publicado em 30/09/2012. 